\title{
Cambios en el modelo de enseñanza de las ciencias del suelo a través de ciclos de mejora en el aula
}

\section{Changes in the teaching approach of soil science through improvement cycles in classroom}

GAEL BÁRCENAS-MORENO

ORCID: https://orcid.org/0000-0002-1329-9967

Universidad de Sevilla

Dpto. Cristalografia, Mineralogía

y Química Agrícola

gbarcenas@us.es

Fecha de recepción: 05-06-2019.

Fecha de aceptación: 06-06-2019.

DOI: http://dx.doi.org/10.12795/9788447221912.007

Pp.: 177-201 


\section{Resumen}

El cambio en el modelo metodológico docente se aplicó a la sección de Edafología (ciencia del suelo) de la asignatura de Edafología y Climatología Agrícola impartida en la Escuela Técnica Superior de Ingeniería Agronómica de la Universidad de Sevilla, mediante la experimentación de dos ciclos de mejora en el aula. En este trabajo se detallan el diseño y los resultados del segundo ciclo desarrollado durante siete sesiones. Se ha partido de la premisa de la necesidad de crear un espacio crítico donde el estudiante se convierta en el protagonista de su propio aprendizaje y el docente abandone su tarima para acompañarle y guiarle en el anclaje de los nuevos contenidos a sus conocimientos previos.

Palabras clave: Edafología, Ingeniería Agrícola, docencia universitaria, experimentación docente universitaria, evaluaciones formativas

\section{Abstract}

The change in teaching methodological model was applied to soil science section of the subject "Soil Science and Agricultural Climatology" which is taught in the Higher Technical School of Agricultural Engineering at Seville University by the achievement of two improvement cycles. In this work are detailed the drawing and the results corresponding to the second improvement cycle which was realized along seven sessions. The premises of the drawing were the creation of a critical space where the students have the leading role while the teacher led them during the learning process.

Keywords: Edaphology, Agricultural Engineering, University teaching; University teaching experimentation, Formative evaluations 


\section{Contexto}

El cambio metodológico a través de ciclos de mejora en el aula (CIMA) se ha llevado a cabo en la asignatura de Edafología y Climatología Agrícola de 1o curso del Grado de Ingeniería Agrícola de la Universidad de Sevilla. Se trata de una asignatura compartida por dos departamentos, de manera que mi departamento (Cristalografia, Mineralogía y Química Agrícola) imparte la docencia correspondiente a Edafología y el departamento de Ciencias Agroforestales se ocupa de la parte de Climatología Agrícola. La parte de Edafología equivale a un total de tres créditos dentro del segundo cuatrimestre, de los cuales dos corresponden a sesiones de teoría y uno a sesiones prácticas en laboratorio.

El primer ciclo de mejora coincidió con el inicio del tema 3 que aparece en el temario de la asignatura como "Compuestos sólidos orgánicos del suelo". Sin embargo, el segundo ciclo de mejora abarcó todo el Bloque III del temario con, un total de 5 temas de los 12 que engloban la parte teórica. En estos temas se tratan las diferentes propiedades del suelo. La correcta comprensión de estos parámetros y las relaciones entre los mismos, son fundamentales para entender el funcionamiento del suelo como ecosistema vivo, dinámico, cambiante y frágil, estableciendo las bases para poder entender procesos de interacción suelo-planta-atmósfera, fundamentales en la ingeniería agrícola.

Jornadas de Formación e Innovación Docente del Profesorado | № 2 (2019) Esta obra se distribuye con la licencia Creative Commons 


\section{Diseño previo del ciclo de mejora en el aula}

\section{Mapa de contenidos}

El mapa engloba los contenidos de los temas 7-11 del temario de la asignatura de Edafología en la que se ha desarrollado el ciclo de mejora. En el mapa (ver Figura 1) ocupa un lugar central la pregunta: ¿Cómo sé yo que mi suelo vale para lo que quiero? Esta se da en el contexto de una situación supuesta donde se le dice al alumnado que imagine que le contrata un cliente al que le han dado una subvención para implementar un cultivo " $X$ " y quiere su asesoramiento para decidir en cuál de sus dos fincas sería mejor hacerlo. En la leyenda aparecen las claves para leer el mapa, que básicamente categoriza el tipo de contenidos mediante las preguntas que vamos a hacer de las diferentes propiedades del suelo a estudiar, ya que el trabajo a realizar por el alumnado debe abarcar el estudio íntegro de estas propiedades desde el punto de vista conceptual, procedimental y actitudinal.

Jornadas de Formación e Innovación Docente del Profesorado | № 2 (2019) Esta obra se distribuye con la licencia Creative Commons Reconocimiento-NoComercial-SinObraDerivada 4.0 Internacional (CC BY-NC-ND 4.0.) 


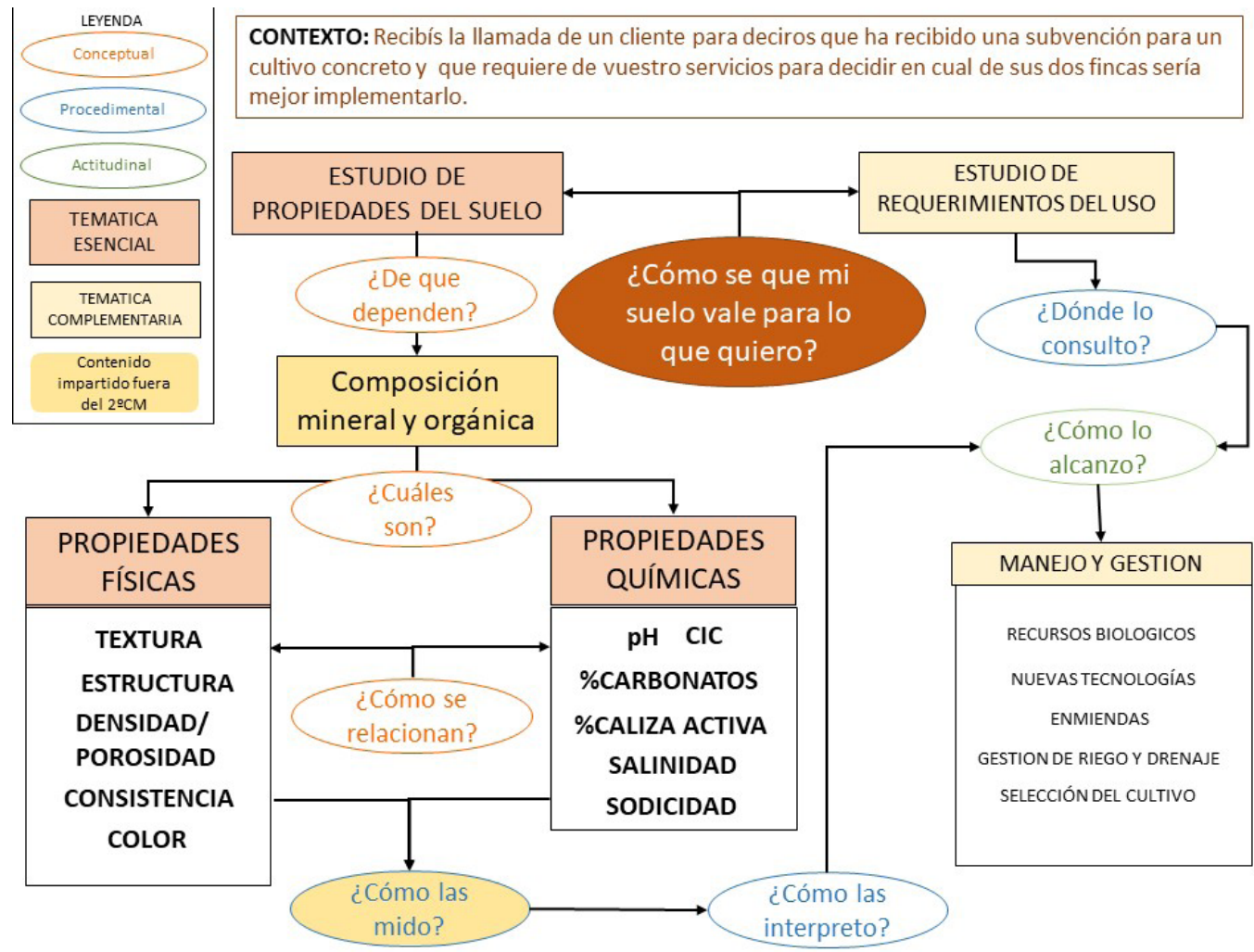

$\mathrm{CM}=$ Ciclo de mejora. $\mathrm{CIC}=$ Capacidad de Intercambio Catiónico

Figura 1. Mapa de contenidos correspondiente al 2o ciclo de mejora

Jornadas de Formación e Innovación Docente del Profesorado I № 2 (2019)

(c) $(1) \Leftrightarrow$ Esta obra se distribuye con la licencia Creative Commons

Internacional (CC BY-NC-ND 4.0.) 


\section{Actualización del modelo metodológico posible}

Tras la aplicación del modelo metodológico posible en un primer ciclo de mejora y observar los inconvenientes, en el segundo ciclo de mejora se aplicó un modelo metodológico con algunas mejoras, que pretenden optimizarlo para su aplicación a un ciclo de mayor duración y relativo a un contenido fundamental de la asignatura.

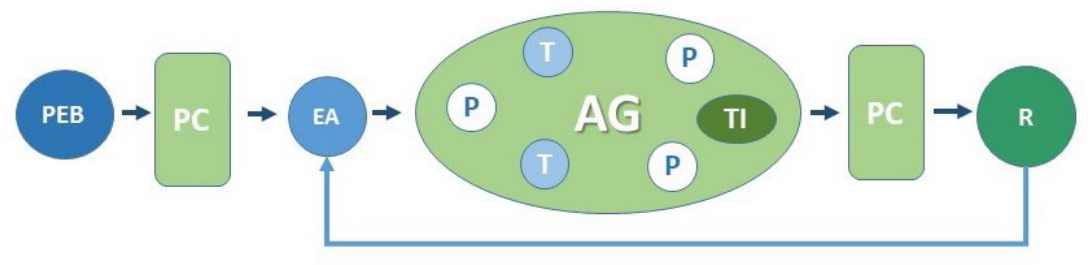

PEB: Planteamiento de una "Pregunta Estímulo Base"; PC=puesta en común; $E A=$ explicación de la actividad en grupo; $T=$ Aclaraciones teóricas; $T$ I=Tutoría entre Iguales; $A G=A c t i v i d a d$ interactiva en grupo; $R=$ Recapitulación colectiva

y explicación de una tarea que responde a un desafio más complejo siguiendo el patrón anterior.

Figura 2. Versión adaptada para el 2o ciclo de mejora del modelo metodológico posible

\section{Secuencia de actividades}

Las actividades diseñadas para este ciclo de mejora siguen el patrón básico de los "talleres conceptuales" propuestos por Finkel (2008), en los que se les proporcionan a los estudiantes los cuatro elementos fundamentales para acompañarles en el proceso de aprendizaje: 1) otra persona con la que compartir el proceso mediante la realización de trabajo en grupo; 2) una secuencia de preguntas bien diseñada que le fuerza a "investigar" y generar hipótesis en torno a un mismo hilo conductor; 3) un profesor al que puede acudir para solventar pequeñas dudas que le obstaculicen el proceso; d) un desenlace intelectual que le permita evaluar los resultados de su trabajo compartiéndolo con sus compañeros y compañeras. 
ACTIVIDAD 1:

Explicación de la dinámica a seguir durante el 2o ciclo de mejora. 5'

Finalidad: Informar al alumnado de la dinámica de trabajo, de las implicaciones en la calificación final y del acuerdo de compromiso de trabajo mutuo.

ACTIVIDAD 2:

Realización del cuestionario inicial. 20'-30'

Descripción: desarrollo de un cuestionario con 6 preguntas plantean situaciones cotidianas donde se ven implicados los contenidos objetivo de este tema y que el alumnado debe responder en base a sus conocimientos previos.

Finalidad: establecer los conocimientos de partida del alumnado y hacerles tomar consciencia de lo que ya saben, de lo que dudan y de lo que desconocen

Contenidos: conocimientos relativos al bloque III del temario. Propiedades del suelo

ACTIVIDAD 3:

Planteamiento de cuestión inicial. 10'

Contexto: Recibís la llamada de un cliente para deciros que ha recibido una subvención para un cultivo concreto y que requiere de vuestros servicios para decidir en cuál de sus dos fincas sería mejor implementarlo.

Pregunta: ¿Cómo sé que mi suelo vale para lo que quiero?

Descripción: se pone a los alumnos en contexto y se les plante la pregunta inicial que servirá de hilo conductor de todo el ciclo

Finalidad: enfrentar al alumnado a una situación familiar con la que se podrían encontrar en su futuro como profesionales para hacerles tomar consciencia de qué herramientas y conocimientos necesitan para afrontar el reto. Contenidos: conocimientos relativos al bloque III del temario. Propiedades del suelo

ACTIVIDAD 4:

Trabajo en grupo 1 “Qué necesita mi cultivo?”. 30”

Descripción: los alumnos se dividen en grupos de 4-5 integrantes, se les asigna un cultivo y deben buscar los requerimientos edáficos del mismo con ayuda del material bibliográfico que le profesor lleva al aula, pudiendo ayudarse de internet.

Finalidad: Conocer diversas fuentes de consulta para asesorarse en sus decisiones, ponerse en contacto con la terminología utilizada en textos técnicos y que identifiquen las diferentes propiedades del suelo que condicionan el establecimiento de los cultivos

Contenidos: Estudio de requerimientos del uso del suelo 
ACTIVIDAD 5:

Puesta en común de los resultados del trabajo en grupo 1 (actividad 4). 20'

Descripción: un portavoz de cada grupo expone lo que ha encontrado sobre su cultivo y se va escribiendo un listado con las diferentes propiedades del suelo que van saliendo a la luz, clasificándolas como fisicas o químicas, comentando las relaciones entre contenidos.

Finalidad: exposición del trabajo realizado por los alumnos y construcción de un listado de propiedades que les haga tomar consciencia de la diversidad de propiedades que hay y la relación entre ellas

Contenidos: Propiedades Físicas y Químicas del suelo

ACTIVIDAD 6:

Trabajo en grupo 2. Estudio de las propiedades del suelo. 90'

Descripción: Partiendo de la lista configurada en la actividad 5, se reparten las propiedades por grupos y se les asigna la tarea de preparar una ficha (aportada por el profesor en blanco) con la información relativa a esa propiedad que debe incluir: ¿Qué es?; ¿Cómo se mide?; ¿Cuáles son sus valores diagnósticos?; ¿De qué factores depende?; ¿Con que otras propiedades se relaciona?; ¿Cuál es su influencia en el suelo? Para ello utilizarán diversas fuentes bibliográficas, tanto en red como impresas que deberán citar adecuadamente.

Finalidad: Que adquieran un conocimiento integral y profundo de las diferentes propiedades, sus formas de medirlo y los valores diagnostico que les servirán de ayuda para interpretar análisis de suelos

Contenidos: Propiedades Físicas y Químicas del suelo

ACTIVDAD 7:

Puesta en común del trabajo realizado en la actividad 6. 60'

Descripción: un portavoz de cada grupo expondrá la ficha completa, mostrado a sus compañeros toda la información encontrada. El profesor irá matizando y corrigiendo si fuese necesario para que la ficha definitiva luego quede disponible para el uso y estudio de tod@s los compañeros.

Finalidad: los alumnos tendrán la oportunidad de ejercer de "profesores" de sus propios compañeros presentando su trabajo y todos podrán obtener una visión global de las propiedades.

Contenidos: Propiedades Físicas y Químicas del suelo 
ACTIVIDAD 8:

Trabajo en grupo 3. Interpretación de análisis. 90'

Descripción: se reparte a los alumnos dos analíticas de suelos diferentes correspondientes a las dos fincas del cliente del contexto de la pregunta inicial. Teniendo en cuenta estos dos informes y el cultivo que se les fue asignado en la actividad 4, deberán realizar un informe donde justifiquen adecuadamente en cuál de los dos suelos sería mejor implantar este cultivo. Y los posibles manejos que podrian realizar para adecuar el suelo al cultivo.

Finalidad: hacer uso de los conocimientos adquiridos hasta el momento en su contexto profesional.

Contenidos: Interpretación de análisis de Propiedades Físicas y Químicas del suelo

ACTIVIDAD 9:

Consultorías entre expertos. 30'

Descripción: se asigna un número a cada integrante de cada grupo y también a los grupos de manera que el alumno cuyo número coincida con el número de su grupo será el portavoz que presentar a los compañeros de otros grupos (que han sido asignados con ese número) los avances realizados en la actividad 8 para obtener su opinión. Mientras, el resto de integrantes de su grupo irán a reunirse con los portavoces de los grupos con su número asignado.

Finalidad: por un lado, hacer que el alumno exponga sus resultados y sus dudas al resto de sus compañeros, a la vez que recibir información y ayuda de los mismos, pero en un ambiente reducido.

Contenidos: Interpretación de análisis de Propiedades Físicas y Químicas del suelo. Manejo y gestión.

ACTIVIDAD 10:

Puesta en común del informe elaborado en la actividad 8. 30'

Descripción: un portavoz de cada grupo expondrá la situación de sus suelos y su cultivo e informará de la decisión que ha tomado y su justificación. Finalidad: cierre de actividad que somete a juicio las decisiones del grupo mediante debate donde podrán revelarse diferentes soluciones a un mismo problema.

ACTIVIDAD 11:

Realización del cuestionario final. 20'-30'

Descripción: desarrollo de un cuestionario con 6 preguntas plantean situaciones cotidianas donde se ven implicados los contenidos objetivo de este tema y que el alumnado debe responder en base a sus conocimientos previos y los adquiridos durante el desarrollo del ciclo.

Finalidad: establecer los conocimientos alcanzados por el alumnado durante el segundo ciclo de mejora y poder realizar las escaleras de aprendizaje. Contenidos: conocimientos relativos al bloque III del temario. Propiedades del suelo 


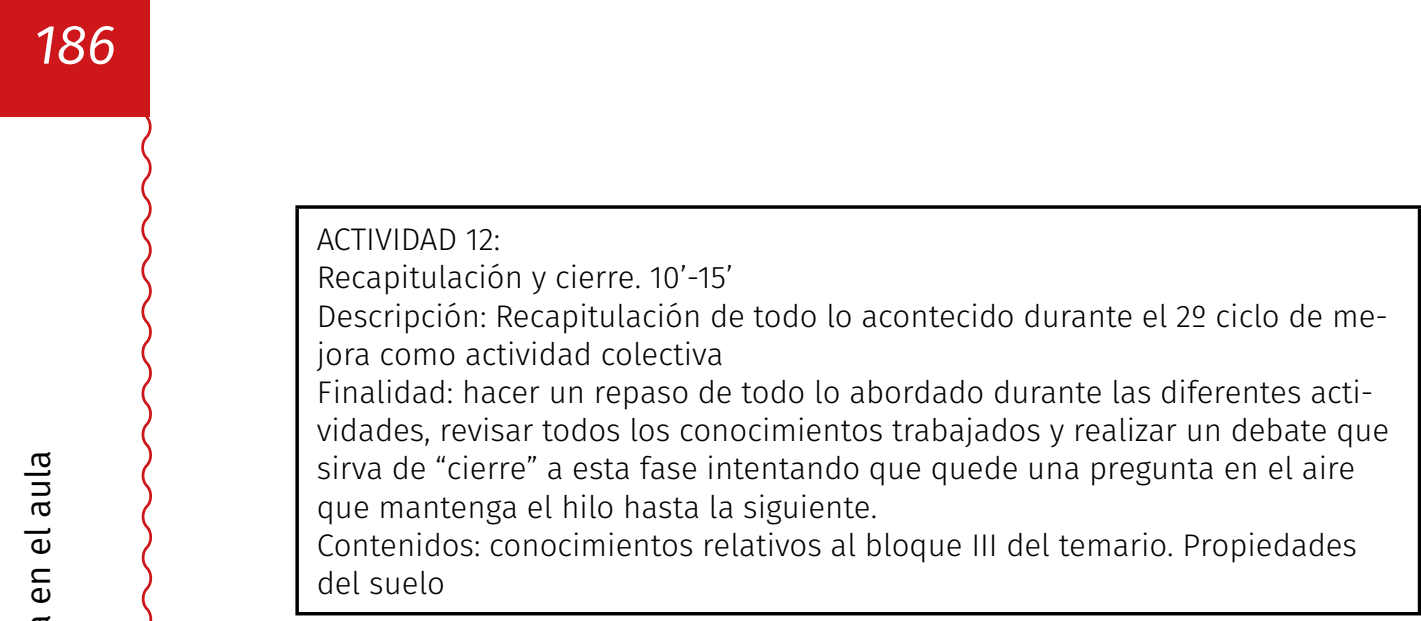

Jornadas de Formación e Innovación Docente del Profesorado | № 2 (2019) Esta obra se distribuye con la licencia Creative Commons Reconocimiento-NoComercial-SinObraDerivada $\quad 4.0$ Internacional (CC BY-NC-ND 4.0.) 


\section{Cuestionario inicial y final}

Como actividad de inicio del ciclo de mejora en el aula se planteó al alumnado la cumplimentación de un cuestionario de exploración de conocimientos previos, con seis preguntas que englobaban de forma general el contenido de los cinco temas a estudiar.

\section{Tabla 2. Preguntas del cuestionario}

Edafología
Cuestionario inicial Bloque de Propiedades del suelo

1) Imagina que te vas de camping y tienes que montar la tienda de campaña, pero se te ha olvidado la maza para clavar las piquetas ¿en qué zona crees que te costaría menos montarla, en la pradera llena de césped o en la zona lisa sin vegetación? ¿Dónde crees que tienes más riesgo de que se te moje la base de la tienda? Explica tu razonamiento

2) ¿Dónde crees que le costaría más trabajo tirar de un carro a una mula, en un suelo arcilloso seco o mojado? ¿y si el suelo fuese arenoso? Explica tu razonamiento.

3) ¿Por qué crees que dentro de un mismo campo de cultivo a veces se ven cambios de color en el suelo a modo de rodales claros y oscuros?

4) ¿Por qué crees que hay plantas que solo crecen en suelos de pH ácidos y otras solo en suelos de $\mathrm{pH}$ más básicos?

5) ¿Por qué crees que, a pesar de haber mucha disponibilidad de agua, en las marismas solo crecen algunas plantas?

6) Imagina que tienes un olivar y tienes la opción de quitar o dejar la vegetación herbácea que crece entre los olivos ¿Dónde crees que tendrá el suelo más capacidad para retener nutrientes, en el caso en el que retiras la vegetación y dejas el suelo al descubierto solo con los olivos, o en el caso en el que dejas las plantas herbáceas en el suelo? Explica tu razonamiento. 


\section{Aplicación del ciclo de mejora en el aula}

\section{Resumen del desarrollo de las sesiones}

Sesión 1: Comienzo explicando la dinámica que vamos a seguir (Actividad 1), mientras dejo tiempo para que lleguen los alumnos, ya en las últimas sesiones ha comenzado a disminuir el número de asistentes, lo que dificulta la previsión de asistencia.

Pasada la prórroga decido asumir que los quince estudiantes que han venido son los que realmente tienen algún tipo de interés en la asignatura y con los que en un principio cuento para hacer el ciclo. Les reparto el cuestionario inicial (Actividad 2) y en torno a los 30 minutos todos lo han entregado, contando con escasos 20 minutos para iniciar la actividad 3, donde les planteo la cuestión de partida. Tras ponerles en contexto, no me dan margen para dejarles pensar un instante, ya que una alumna de las más motivadas da una respuesta contundente nada más escuchar el planteamiento y todos se lanzan a dar sus opiniones, que realmente son bastante obvias y comunes. Esto me hace cuestionarme si no debería haber planteado otra cuestión que entrañase algo más de complejidad, aunque realmente la función de motivación de la pregunta sí se ha cumplido, porque parecen muy interesados en el planteamiento de este pequeño reto donde ellos/as serán los protagonistas.

Teniendo en cuenta la inercia que ha cogido el grupo decido adelantar con la siguiente actividad, aunque solo sea para plantearla y que puedan empezar a trabajar formando los grupos, La primera actividad en grupo (Actividad 4) se centra en responder a la pregunta “¿Qué necesita mi cultivo?", de manera que les reparto a cada grupo un cultivo diferente y les dejo material bibliográfico para que puedan empezar a buscar información. Al finalizar la 
sesión, prácticamente todos los grupos han encontrado información y dejamos pendiente para la segunda sesión finalizar esta tarea.

Sesión 2: Me marcho a clase cargado de libros y cuando llego y veo que hay aún menos estudiantes que el día anterior, habiendo grupos que ni tan siquiera tienen algún representante para trabajar. Les dejo la primera parte de la clase para que terminen la tarea iniciada el día anterior y para que preparen la puesta en común de lo que han encontrado (Actividad 5), donde analizaremos entre todos qué tipos de factores y propiedades suelen limitar o condicionar de alguna manera el desarrollo de los cultivos.

La puesta en común da mucho juego, enfrentarse a los compañeros e intentar dar sus explicaciones evidencia muchas de sus limitaciones y la confusión de términos y conceptos que tienen. Esto, por otro lado, desafia mi capacidad para ir aprovechando esas pequeñas fisuras, para ir rellenando y creando una base buena para todos/as, sin que el estudiante se sienta corregido de forma negativa, intentando que sean sus propios compañeros los que vayan guiándole y animándoles a que se esfuercen en buscar la mejor manera para expresar esa idea difusa que tienen en la cabeza. A veces, un concepto, un nombre, bloquea el proceso y es sorprendente observar cómo una vez que ese pequeño bache se supera, fluyen enlazando conceptos y reforzando lo que tímidamente van construyendo.

Sesión 3: El día anterior dejamos pendiente terminar la puesta en común correspondiente a la actividad 5 , pero al volver a encontrar el aula medio vacía me es imposible continuar con esa actividad, ya que el grupo que tenía que terminar de exponer su parte no ha venido. Dado que los asistentes están totalmente descentrados pensando en el examen de otra asignatura que tienen más tarde, decido presentarles la siguiente tarea en la que deben trabajar 
y que ellos/as elijan cómo gestionar su tiempo para trabajar, en clase o en casa. De este modo tan improvisado y poco alentador presento la actividad 6, donde afrontarán el estudio de las diferentes propiedades del suelo, haciéndose cargo cada uno de los grupos de trabajar un conjunto de actividades concretas, de las que tendrán que elaborar unas fichas y que, posteriormente, nos servirán a todos como fichas resumen que pueden ser muy útiles para afrontar la siguiente actividad y para preparar el examen (previamente corregidas por mi). Sorprendentemente y contra todo pronóstico, se ponen a trabajar en las fichas.

Al finalizar la sesión me queda una sensación agradable al pensar que, si hoy hubiese sido una clase tradicional, ninguno de los asistentes se habría enterado de nada, porque mientras yo hubiese estado haciendo la exposición sobre el tema correspondiente, ellos habrían estado mirando sus apuntes, totalmente ausentes, mientras que hoy, teniendo la opción de estudiar, han preferido trabajar en la asignatura.

Sesión 4: Hoy estamos algunos más en clase, ha reaparecido uno de los grupos que había faltado y mientras los otros siguen afanados en la actividad 6, pongo al día al grupo rezagado, mientras voy atendiendo las diversas dudas que van surgiendo a los demás.

Es el último día antes de las vacaciones de Semana Santa y veo adecuado dar cierre a esta actividad para iniciar la 7 a la vuelta. Para ello debo reconducir un poco mi planteamiento inicial y les pido que me envien las fichas antes de finalizar las vacaciones, para que yo pueda corregirlas y enviarles las versiones corregidas.

Sesión 5: Tras preparar todo el material necesario para que iniciemos hoy la Actividad 8, les entrego en un sobre a cada grupo dos análisis de suelos diferentes, de manera 
que deberán evaluar cuál de los dos suelos es más óptimo para el cultivo que se les asignó en un principio, y para el cual realizaron un estudio de los requerimientos edáficos con la actividad 4. Para poder interpretar y valorar los análisis de suelos podrán ayudarse de las fichas que han elaborado durante la actividad 6 y que ya tienen corregidas. En previsión de que después de Semana Santa pudieran reincorporarse algunos de los grupos de los que desaparecieron al inicio del ciclo, preparo material extra.

Una vez entregada toda la documentación, se ponen muy decididos a trabajar, cada grupo a su ritmo, algún grupo me pide usar el ordenador para ir haciendo directamente el informe, mientras otros se ven aun algo abrumados haciendo el análisis de suelos.

Conforme van surgiendo las dudas me doy cuenta de que he ido tan desbordado de trabajo que no he establecido ningún patrón fijo para el informe y tengo que ir dándoles instrucciones no del todo pensadas y organizadas. Es este un inconveniente, consecuencia del exceso de trabajo. Indudablemente, de los errores se aprende, y tengo la certeza de que este es un proceso en constante cambio y evolución, sin embargo, no puedo evitar que en estos momentos la sombra del examen al que tendrán que someterse oscurezca mi ánimo momentáneamente.

Sesión 6: Hoy la previsión es seguir trabajando, pero para compensar la falta de especificaciones que les di el lunes para elaborar el informe, he creado unas instrucciones para que entreguen los mismos apartados, asegurándome de que trabajan los contenidos como considero que les va a ayudar más. Al redactar las instrucciones me doy cuenta de mi propio criterio, y de que realmente todo este trabajo solo tiene sentido y eficacia si ellos se comprometen a trabajar también. Los apartados que deben completar son un repaso completo por los contenidos, 
pero aplicado a lo procedimental, es decir, a la elaboración de un informe justificando su decisión como ingenieros. Creo que les ayudará a tomar consciencia de su responsabilidad.

Al llegar observo con una mezcla de alegría y tristeza que la clase ha vuelto a llenarse, una pena cuando ya es la penúltima sesión y un problema a la hora de trabajar en los grupos. Los alumnos que aparecen y desaparecen, no son compatibles con esta forma de trabajo y al terminar la sesión me doy cuenta de que no solo es incómodo para mí, sino que es un gran contratiempo para los que vienen todos los días a clase y llevan todo el cuatrimestre trabajando. Este es otro factor a tener en cuenta con este formato de trabajo, y tomo consciencia de la importancia del tema del compromiso del que hablamos en el curso de docencia universitaria. Sin duda, de adoptar esta metodología para el total de la asignatura desde el inicio del curso, sería de vital importancia dejar muy claro el compromiso de estudiantes y profesores, tener muy claro los criterios de evaluación y calificación para que los conociesen desde el inicio con total claridad. Sería también muy importante intentar que en cada bloque de contenidos haya un trabajo en grupo y otro individual, aunque como siempre surge la duda del tiempo que requiere esta evaluación por parte del docente. Creo que, en este caso, la utilización de las TICS y la posibilidad de realizar autoevaluaciones podría resultar una alternativa en algunas ocasiones en las que se centre mucho el trabajo en grupo, y de hecho será lo que haga para compensar esta situación con los alumnos/as "intermitentes", además de la asistencia.

Sesion 7: Hoy es nuestra última sesión y, entre la realización de las encuestas de la Universidad de Sevilla, el cuestionario final y un cuestionario para registrar la opinión del alumnado sobre el cambio metodológico 
experimentado durante el segundo ciclo de mejora, apenas queda tiempo para hacer una actividad de cierre. Comienzo atendiendo dudas sobre el informe que deben entregar, y poco a poco va llegando el alumnado.

Mientras terminan de rellenar las encuestas decido proyectar el mapa de contenidos para que me ayude a hacer un cierre de la actividad, explicándoles qué es y refrescándoles la memoria de todo lo que han hecho durante el desarrollo de este ciclo. Una vez concluido el resumen de la actividad y el intercambio de opiniones, me veo forzado a interrumpir para pasar a entregarles los diversos cuestionarios.

\section{Análisis comparativo del cuestionario inicial y final}

El cuestionario inicial/final, descrito con anterioridad, constaba de preguntas relacionadas con los contenidos que se iban a trabajar durante el segundo ciclo de mejora, pero hay que indicar que estos contenidos no se han trabajado contestando literalmente a las preguntas del cuestionario, sino que se han abordado mediante la realización de actividades que atendían a la pregunta estimulo inicial (que no aparece en el cuestionario).

El cuestionario inicial lo cumplimentaron 18 estudiantes, mientras que el cuestionario final -con las mismas preguntas- lo respondieron 14 , coincidiendo en ambas ediciones solo 11 estudiantes, cuyas respuestas han podido ser comparadas.

\section{Análisis de los resultados}

En la figura 3 podemos ver un resumen de los resultados obtenidos al analizar la frecuencia de respuesta en 
cada nivel, tanto en el cuestionario inicial como final, para cada una de las preguntas detalladas en la parte inferior.

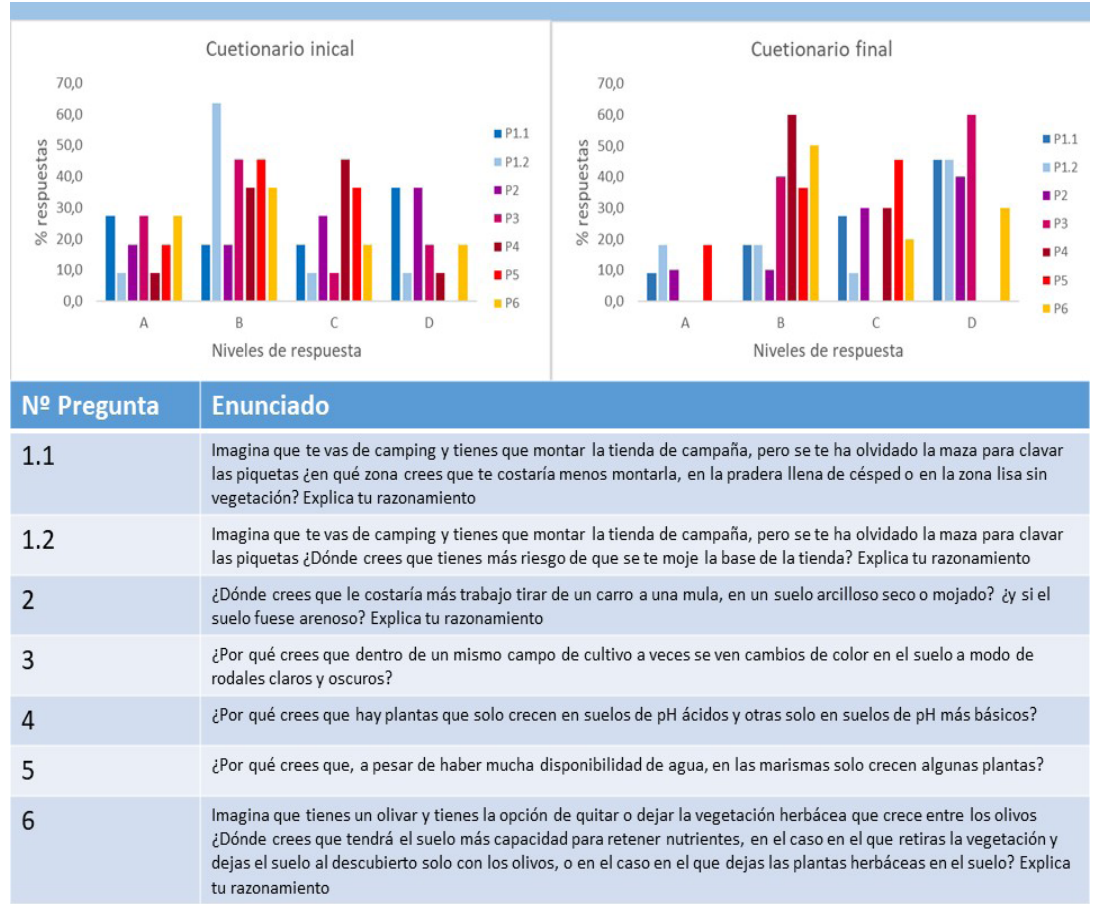

Figura 3. Resumen de los resultados obtenidos en los cuestionarios inicial y final

Los resultados obtenidos en el cuestionario inicial P1.1 muestran una gran diversidad de niveles en el aula, aunque el porcentaje sea algo mayor en el nivel más bajo, todos los niveles se ven representados. En el cuestionario final, aunque la tendencia no es evidente, sí se observa una importante disminución de respuestas en el nivel más bajo, que se ven repartidas en el resto de los niveles quedando el nivel medio y el más elevado con el mismo porcentaje de respuestas.

En los resultados del cuestionario inicial P1.2 se observa un elevado porcentaje de la clase en el nivel $B$, se percibe que la relación vegetación/humedad la tienen clara, pero el papel del suelo queda totalmente fuera de 
plano. Sin embargo, en el cuestionario final P1.2 el porcentaje más alto se centra ahora en el nivel "D", lo que sugiere la incorporación del suelo a sus razonamientos. También se observa un incremento en el nivel más bajo, debido a la ausencia de explicación y argumentación de sus respuestas, que podría deberse tanto a la apatía de responder dos veces a la misma pregunta, como a la falta de asentamiento de toda la información recibida durante el ciclo de mejora.

En el caso de la pregunta 2 del cuestionario inicial, los porcentajes están más o menos repartidos en todos los niveles, con algo más de protagonismo en los niveles más altos, lo que evidencia probablemente un conocimiento previo anclado a la experiencia propia. Los cambios de P2 en el cuestionario final son poco marcados, ya que partíamos de unos resultados del cuestionario inicial con niveles altos, sin embargo, sí se aprecia que la mayoría de los estudiantes se establecen en los niveles más altos después del ciclo.

Los resultados del cuestionario inicial en P3 sitúan a la mayoría de la clase en los primeros niveles de conocimiento establecidos, evidenciando que se conoce esa realidad, pero se tiene una idea muy limitada de su origen. En el cuestionario final se reparten todos los estudiantes en dos escalones: el "B" y el "D"; en ambos casos se concretan ejemplos, pero los del nivel intermedio siguen teniendo una idea limitada del suelo y de los procesos que en él se desarrollan.

En el cuestionario inicial de $\mathrm{P} 4$ se reparten los resultados entre los escalones intermedios, con un nivel bastante alto. Por el contrario, en el cuestionario final, aunque se concentran aún más los resultados en estos niveles, parece haber una tendencia a simplificar las respuestas, no llegando ningún alumno al nivel más alto, ni tan siquiera el que lo obtuvo en el cuestionario inicial. Esto evidencia, 
en mi opinión, un fallo de diseño al repetir las preguntas, ya que parece que el alumnado no entra en detalles al repetir el cuestionario, y estos detalles son claves a la hora de valorar hasta dónde llega su comprensión de la complejidad del suelo.

En P5 solo se han detectado tres niveles. En el cuestionario inicial la mayor parte de respuestas estaban en el nivel intermedio, con elevada participación también en el nivel superior. En el cuestionario final solamente hay un pequeño desplazamiento del nivel " $B$ " al " $C$ ".

Las respuestas de los estudiantes en el cuestionario inicial de P6 se concentran en los dos primeros niveles, existiendo un componente evidente del perfil de ingeniero agrícola en la visión de la competencia de la vegetación herbácea con el cultivo (olivar). En el cuestionario final aparece una concentración de respuestas elevada en el nivel "B" al que parecen haberse sumado parte de los que había en el nivel "A" e, incluso, algunos de niveles superiores, existiendo solo un pequeño aumento en los niveles más altos. Posiblemente el enfoque de la última parte del ciclo hacia un análisis de suelos y la toma de decisiones sobre la idoneidad de un cultivo, haya hecho que en las respuestas se priorice la competencia inmediata entre especies, en vez de realizar una respuesta enfocada a la capacidad de retener nutrientes por parte del suelo. Esta tendencia podría deberse, por un lado, a la ya mencionada apatía/prisa por acabar -al realizar tantos cuestionarios el mismo día-, como a la falta de comprensión del enunciado o a la falta de atención al leerlo, ya que solo aquellos estudiantes que se sitúan en los niveles " $D$ " contestaban realmente sobre la retención de nutrientes por parte del suelo. 


\section{Valoración de los resultados}

En general los resultados reflejados por las escaleras no son nada alentadores, si bien existen varios factores que podrían ayudar a atenuar algo la sensación negativa que deja ver un empeoramiento generalizado de las respuestas de los estudiantes tras el ciclo.

En primer lugar, podemos realizar un repaso por el factor estudiante y sus circunstancias, muchos de ellos han asistido de forma intermitente a clase y el ciclo se ha cerrado tras un par de sesiones después de las vacaciones de semana santa. El empeoramiento de ciertas respuestas viene dado por el hecho de que sean las mismas preguntas, ya que muchos han bajado de nivel al contestar con menos detalle que la primera vez, no porque hayan contestado mal. Esto nos lleva a insistir en cuestionar el hecho de que las preguntas sean exactamente las mismas en el cuestionario inicial y en el final.

Otro factor a tener en cuenta es que los estudiantes no han trabajado durante el ciclo esas preguntas de forma específica, sino que han trabajado las propiedades del suelo que podrían ser las respuestas a esas preguntas. En este caso, mi reflexión me lleva a pensar que más que plegar totalmente al lenguaje y situaciones cotidianas por encima de todo, hay que saber elegir muy bien las cuestiones para que nos permitan, no solo detectar los obstáculos para poder ayudarlos a superarlos con la realización del ciclo, sino también diseñar preguntas de tal manera que realmente podamos valorar si los obstáculos han sido superados.

Sin duda a la lista de factores hay que sumar mi propia inexperiencia a la hora de llevar el ciclo y todos sus pasos. A pesar de todo, se observan saltos de nivel positivos, sobre todo en los estudiantes que iniciaron el ciclo en los escalones más bajos, aunque se puede detectar algún 
individuo que apenas experimenta cambios, evidenciando algún problema más profundo de aprendizaje y/o comprensión de los contenidos.

Sin duda, una vez optimizados los cuestionarios, no hay lugar a dudas de que se trata de una herramienta de evaluación del aprendizaje muy práctica, que cumple la doble función de permitirnos detectar los niveles de partida y los obstáculos, permitiendo además de un seguimiento personalizado de cada alumno, una adaptación al grupo para un proceso correcto y fructífero de aprendizaje.

\section{Evaluación del ciclo de mejora en el aula}

Con la realización del curso, uno de los primeros cambios en mi opinión más transcendentales e irreversibles, fue el cambio en la jerarquía de los roles alumnado/profesorado. El hecho de focalizar como protagonista en las clases al que realmente es objeto del aprendizaje es uno de los cambios más lógicos y, sin embargo, el menos usual, que sin duda formará parte de mi modelo didáctico personal de futuro. He podido comprobar que los estudiantes, a pesar de parecer reacios a estos cambios por falta de costumbre, una vez que se familiarizan con ellos, los reclaman y se muestran apáticos ante una vuelta al sistema tradicional de clase magistral (salvo en algunas cuestiones para las que precisan una guía para poder seguir avanzando en su propio aprendizaje).

Este cambio de roles, no solo les ayuda a aprender de forma más eficaz y a una mayor proporción de alumnado, sino que les hace las clases amenas, despierta su interés y curiosidad y fomenta la confianza en sí mismos, capacitándolos con un gran abanico de competencias necesarias para el desarrollo de su labor profesional.

La categorización de los tipos de contenidos ha sido para mí una aportación muy importante, ya que como 
Biólogo soy amante de llamar a las cosas por su nombre para clasificarlas, y el hecho de llegar a distinguir y poder clasificar los tipos de contenidos mediante la elaboración del mapa de contenidos ha sido un trabajo duro, pero finalmente muy gratificante y necesario (Frega, 2015). Esta tarea ha sido dificil porque te obliga a tomar consciencia y cuestionarte todos los contenidos que llevamos en los programas "heredados" de unos cursos a otros y que, por recomendaciones de colegas de los departamentos y centros, tienden a cambiar lo menos posible de unos años a otros, a pesar de haber sufrido cambios muy importantes en las titulaciones y en el mundo laboral al que se enfrentan ahora los estudiantes del futuro.

Sin duda, la elaboración de los mapas de contenidos es una herramienta muy útil para organizar tanto el temario completo de una asignatura, como un tema concreto. Para el curso próximo realizaré este tipo de mapas con la asignatura completa, para que me sirvan de referencia a mí y a los alumnos.

Mi modelo metodológico personal ha sufrido bastantes trasformaciones, y me temo que aún tendrá que seguir evolucionando para adaptarse no solo a lo deseable, sino también a lo posible, que por desgracia no es una constante en el tiempo. Por ejemplo, el absentismo en clase en determinados momentos del cuatrimestre cuando los parciales de otras asignaturas se concentran hacen inviable ciertas partes del modelo que aún tengo que ver cómo solventarlas, principalmente las relacionadas con los trabajos en grupo y la puesta en común de resultados. Teniendo en cuenta la experiencia de este curso, mi idea es continuar con un modelo base igual para todo el curso partiendo de las premisas de la creación de un ambiente crítico natural acorde a los criterios de Bain (2005), aunque probablemente amolde el tipo de actividades y la secuencia de las mismas para ir salvando los inconvenientes que se generan a veces y que, aunque no dependen de 
nosotros, siempre siguen una pauta más o menos predecible en el tiempo. Otra opción que estoy barajando es la de consensuar con los alumnos desde el principio el modelo y la dinámica y decidir con ellos un calendario previo de actividades para que puedan organizarse con tiempo, pero esto va a requerir de un trabajo intensivo por mi parte a la hora de preparar la asignatura que, por el momento, no sé si seguiré impartiendo el año que viene.

Además de los cuestionarios, la realización de tareas individuales y en grupo, permiten detectar errores comunes a la hora de captar los conceptos, haciendo de gran utilidad estas formas de evaluación formativa, aunque como punto "negativo" hay que argumentar que solo son válidas si el docente es capaz de encontrar tiempo y energía para generar una corrección / feedback rápido que le permita redirigir el proceso de aprendizaje (Gibbs y Simpson, 2005). Por ello creo que hay que economizar y diseñar muy bien tanto el momento y composición de los cuestionarios como la realización de tareas y actividades. Las puestas en común son un sistema rápido y eficaz de evaluación formativa, donde, además, la resolución de un obstáculo de un alumno en particular puede ayudar a los demás; en ocasiones son ellos mismos quienes se ayudan sin necesidad de que lo haga el profesor.

De forma adicional, he de decir que el hecho de llevar un diario del profesor (Porlán, 2008) puede ser una herramienta muy útil para llevar un registro de lo que ocurre en clase para poder ir optimizando el proceso y, el hecho de forzarse a escribir, nos obliga en cierto modo a tener que hacer un análisis de lo ocurrido en clase, que, si no se escribe, a veces se diluye en el maremágnum de cosas que nos ocupan. 


\section{Referencias bibliográficas}

Bain, K. (2005). Lo que hacen los mejores profesores universitarios. Valencia: Universidad de Valencia.

Finkel, D. (2008). Dar clases con la boca cerrada. Valencia: Publicaciones Universidad de Valencia

Frega, D.J. (2015). Recorte, selección y organización de contenidos. Escritos en la Facultad, 109, 13-15.

Gibbs, G. y Simpson, C. (2005). Conditions Under Which Assessment Supports Students' Learning. Learning and Teaching in Higher Education,1, 331.

Porlán, R. (2008). El diario de clase y el análisis de la práctica. Averroes: Red telemática educativa de Andalucía.

Porlán, R. (Coord.) (2017). Enseñanza Universitaria. Como mejorarla. Madrid: Morata/ Ediciones. Universidad de Sevilla. 